\title{
Study on Collaborative Product Development and Digital Engineering Tools
}

\author{
Patrick Müller ${ }^{1, *}$, Florian Pasch ${ }^{1}$, Roland Drewinski ${ }^{2}$, \\ Heinz Bedenbender ${ }^{3}$, Haygazun Hayka ${ }^{1}$, and Rainer Stark ${ }^{1}$ \\ ${ }^{1}$ Fraunhofer IPK, Berlin, Germany \\ \{patrick.mueller, florian.pasch, haygazun.hayka, \\ rainer.stark\} @ipk. fraunhofer.de \\ ${ }^{2}$ PLM Vendor, Germany \\ rdecontact.de \\ ${ }^{3}$ VDI, Germany \\ bedenbender@vdi.de
}

\begin{abstract}
Today, product development is characterized by cross-company collaboration and an intensive exchange of product data. Engineers typically apply a multitude of digital engineering tools and act in diverse engineering processes. In a current study, the "culture" of today's collaborative product development was investigated in significant detail. The questions are: How do engineers work today? Which tasks play a major role in their daily business? How much time is remaining for core engineering tasks like design, calculation and design validation? How do engineers assess their workplace (environment)? Where are opportunities for enhancement of processes and digital engineering tools? In total, 1,401 engineers answered the questionnaires. Details of the study and selected findings are presented in this paper. Interesting findings are related to collaborative engineering and engineering design, Product Data and Lifecycle Management (PDM/PLM), virtual product creation, information and process management, and new media in engineering design.
\end{abstract}

Keywords: Collaborative Engineering, Product Data and Lifecycle Management (PDM/PLM), Digital Engineering Tools, Virtual Product Creation, Process Standards, Engineering Efficiency, new Media in Engineering.

\section{Introduction}

Product creation is facing the next level of fundamental changes and severe challenges in terms of collaboration and information management. Global demands are growing substantially to achieve energy efficient and sustainable value creation networks for production, products, and services without compromising traditional success factors such as time to market, cost, and quality.

In order to stay competitive under such conditions, collaboration partners in industry and public sectors will require new interplay solutions for engineering design

\footnotetext{
* Corresponding author.
} 
execution, domain knowledge representation, expert competence utilization, and digital assistance systems; for further details see [1].

Product development is characterized by cross-company collaboration and an intensive exchange of product data. Engineers typically apply a multitude of digital engineering tools and act in diverse engineering processes. Finally, efficient collaboration amongst companies and disciplines is a key for successful engineering of complex systems, variant-rich systems, and customized products and needs for efficient application of digital engineering tools. Process standards on the one hand and tools for virtual product creation on the other hand play a major role in engineering practice. Product Data and Lifecycle Management (PDM/PLM) and process standards are central means to enable and control information exchange amongst (internal and external) collaboration partners. Anyhow, the supply chain and engineering competencies are distributed in terms of location, discipline, etc.

Finally, the product development process cannot be carried out by individual product developers [2-6]. There is a growing need for a collaborative product development process. This allows collaboration across domains, organizational boundaries and product development phases including a consistent consideration of customer requirements and restrictions from the entire product life cycle [2, 6, 7]. Collaboration capability across individuals, teams, and organizations becomes a key engineering work skill in both directions: horizontally along the phases of the PDP (Product Development Process); vertically along the supply chain and between disciplines. Figure 1 is summarizing such aspects.

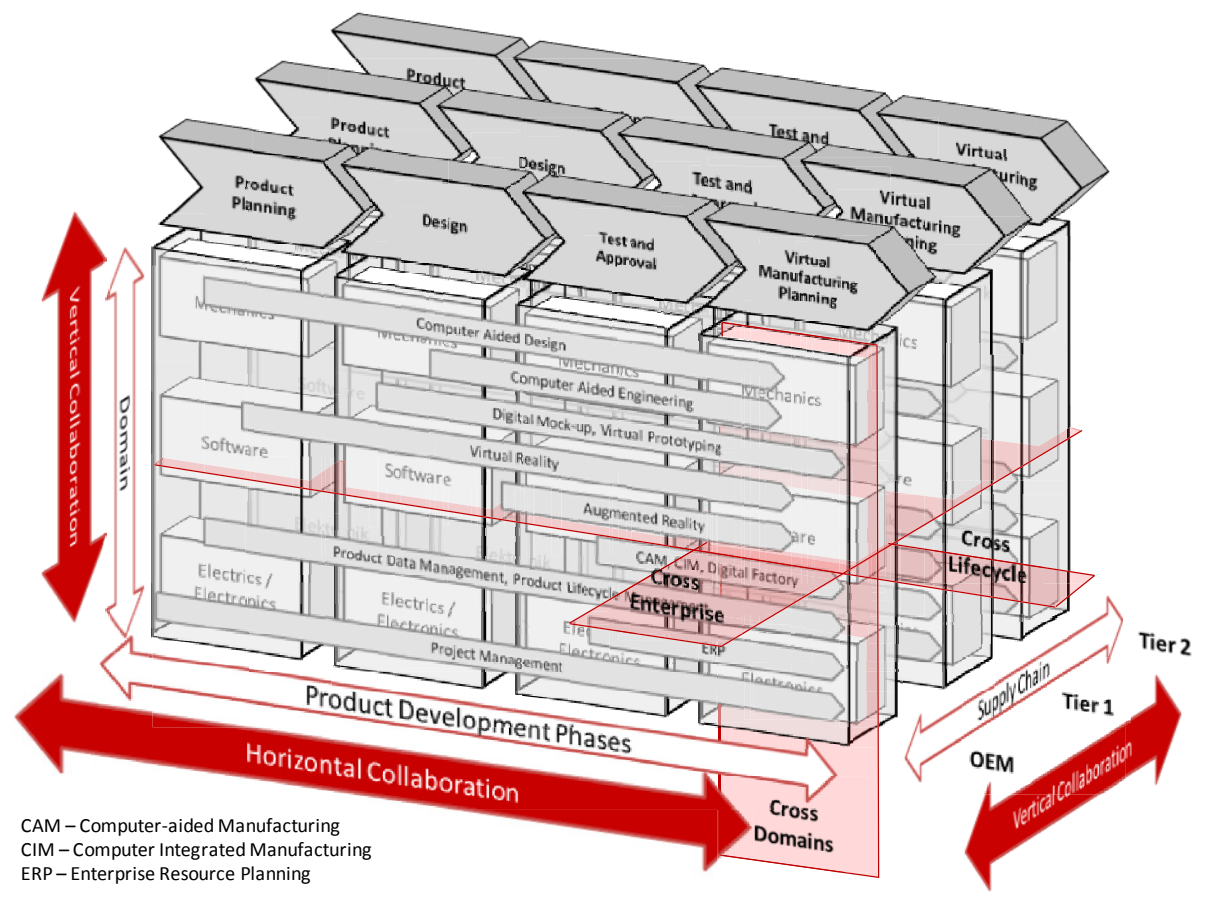

Fig. 1. Complexity in Product Development Processes and Engineering Collaboration 
This scenario is challenging for engineers performing their daily tasks and for vendors of virtual product creation tools at the same time. To analyze this setup from a neutral and scientific perspective a "Study on collaborative product development and digital engineering tools" has been carried out in German industry by three partners: Firstly, the Division Virtual Product Creation of the Fraunhofer Institute for Production Systems and Design Technology IPK (Berlin, Germany), a research institution. Secondly, CONTACT Software (Bremen, Germany), a PLM solution vendor. Thirdly, the VDI (Verein Deutscher Ingenieure, Düsseldorf, Germany), an important engineers' association.

Within the study, the "culture" of today's collaborative product development is investigated in detail. The target of this study is to gain deep insight into dependencies of collaboration influencing factors, to develop a model to assess collaboration in industrial product creation processes and to derive indication for improvement of digital engineering and collaboration tools.

\section{Capabilities and Strategies in Industry}

\subsection{Product Creation and Collaboration Capability in Industry}

From a business perspective, collaboration capability is an issue of competitiveness, as explained above. Nevertheless, so far, there is hardly any model to assess collaboration capability as such, assessing internal and external collaboration processes and tool usage. Similar is the situation for product creation in particular. There is no standard certificate for product development process (PDP) capability, which is suitable to assess reliability, repeatability, innovation strength, and compatibility with standard processes as known form quality management assessments. Particular models for process maturity assessment do exist - for instance $\mathrm{CMMI}^{1}$ or $\mathrm{SAGA}^{2}$ - but these just apply to a subset of aspects of PDP capability and IT use.

From an engineer's perspective, the feeling is dominating that there is a lack of time to concentrate on the main development task, which is to design and validate new systems or system components. Standard processes, tools, techniques etc. request much concentration / attention and "consume" huge amounts of working time.

The coordination of modern development projects is increasingly repressing technically sound design work. Digital engineering tools and PLM solutions for most users are technically and functionally too complex. This dilemma is becoming a serious risk, which just few top managers consider to the necessary extent. Virtual product creation needs stepwise extendable PLM solutions, in order to support the daily work of various disciplines.

\subsection{PLM Vision and Strategies in Industry}

Valuable studies on PDM/PLM have recently been carried out, for instance by Abramovici et al. [8] or the RAAD Research unit of Hoppenstedt [9]: Abramovici et al. [8] for instance investigated the trends of lifecycle management solutions until 2020 in a

\footnotetext{
${ }^{1}$ Capability Maturity Model Integration.

${ }^{2}$ Standards and Architectures for eGovernment Applications.
} 
study involving 47 PLM experts. The focus is on PLM solutions, but not on the subtleties of collaboration. Anyhow, according to their data $71 \%$ of the experts agreed that PLM will establish as the central integration platform for engineering data, processes, and applications until 2020. Approximately two third of the experts suggested that PLM solutions will be independent solutions while one third thinks that PLM will be integrated element of corporate (company-spanning) ERP and Supply Chain solutions. Almost all experts (96\%) expected that PLM solutions in 2020 will increasingly support cross-domain and cross-company engineering cooperation. Parameter-driven optimization of engineering-processes, PLM supported planning of engineering processes, and information analysis and visualization for top managers are expected becoming core functionality of PLM solutions (all awaited by more than two thirds of the experts). Finally, the amount of PDM functionality and use will rise not just for the management of virtual product data but also for data of installed systems over their entire lifecycle. RAAD [9] investigated the situation in 227 companies with more than 500 employees with an inquiry under managing engineers in engineering, production, and IT departments. The majority of companies were related to mechanical engineering and metal machining. RAAD found out that $66 \%$ (of $n=193$ ) had no dedicated PLM strategy, which was committed with the company management. Just $22 \%$ have a strategy, which involves all units. 37\% (of $n=213$ ) said that PLM has a high or very high relevance in their companies.

Both studies show that PLM is a future-oriented topic. Nevertheless, consolidated PLM strategies still are lacking in most even large companies. Anyhow, both studies do not address engineering practice in detail.

\section{Study on Collaborative Product Development and Digital Engineering Tools}

To understand the very nature and culture of collaboration in industry, to update facts and figures on engineers' daily schedules and finally to calibrate ideas on process and collaboration improvement, a study has been carried out in Germany at the end of 2011. This study is complementary to those mentioned above. The premise of this study was an explicit scientific orientation, which was required by all three partners. Firstly, a comparably high number of answered questionnaires was required in order to have a resilient set of up-to-date data. Secondly, there was explicitly no focus on IT systems or solutions of selected vendors. Thirdly, end users have been asked without any channel through company mailing lists or filters.

\subsection{Study Design and Execution}

The study was executed in two major phases: One phase for a "pre-study" based on telephone interviews, one phase for the "core study" applying web-based questionnaires. The following paragraphs provide insight into the major research questions and details of the two phases. Figure 2 briefly summarizes facts of the study design. 


\section{Research Questions}

The core research questions of the study are the following:

- How do engineers work today?

- Which tasks play a major role in their daily business?

- How much time is remaining for core engineering tasks like design, calculation and design validation?

- How do engineers assess their workplace (environment)?

- What are promising future technologies to enhance collaboration in industry?

\section{Questions Blocks}

These questions have been broken down into the following question blocks, which contained different sub-questions and response items:

1. Questions on the company (branch, size, competencies, supply chain position)

2. Questions on the tasks of the respondent (education, discipline, position, main engineering tasks)

3. Questions on development activities (PDM and ERP usage, workplace location and time spent in each location, amount of time spent on creative work, formal tasks etc.)

4. Questions on collaboration (time spent on internal and external product data exchange; technical environments used for data exchange; assessment of work environment such as tool and facility availability, assessment of coordination, communication, knowledge integration and information logistics)

5. Question on "future-oriented" ideas and permanent issues

Findings are related to collaborative engineering and engineering design, Product Data and Lifecycle Management (PDM/PLM), virtual product creation, information and process management, and new media in engineering design.

\section{Pre-study: Interview Study}

In order to "calibrate" the questionnaire study, an interview study with eight companies from German industry (automotive, plant construction, chemical industries, energy systems, engineering service provider) was undertaken in advance. Each interview was carried out via telephone and took between one and one and a half hour. The participants have been engineers (development experts, (technical) project leaders, managing IT engineers (responsible for PDM/PLM, CAx, ERP)). This pre-study was vital to calibrate the own terminology and to understand, if own suggestions were going into the right direction. Interesting is that the small number of cases brought results drawing a parallel to the results of the large questionnaire study (see section 4).

\section{Core Study: Web-Based Questionnaire (Online Inquiry)}

The link to a web-based questionnaire was mailed to 22,000 engineers in Germany being members of a large German engineers association, namely the VDI. All receivers were personally asked "addressing their engineering background and work in general" and not their work affiliated to a particular employer. This was necessary to lower confidentiality obstacles. The response rate was higher than $6 \%$, which enabled a total sum of 1,401 engineers having participated. The elicitation was carried out from end of October to the mid of November (3 weeks) in 2011. 

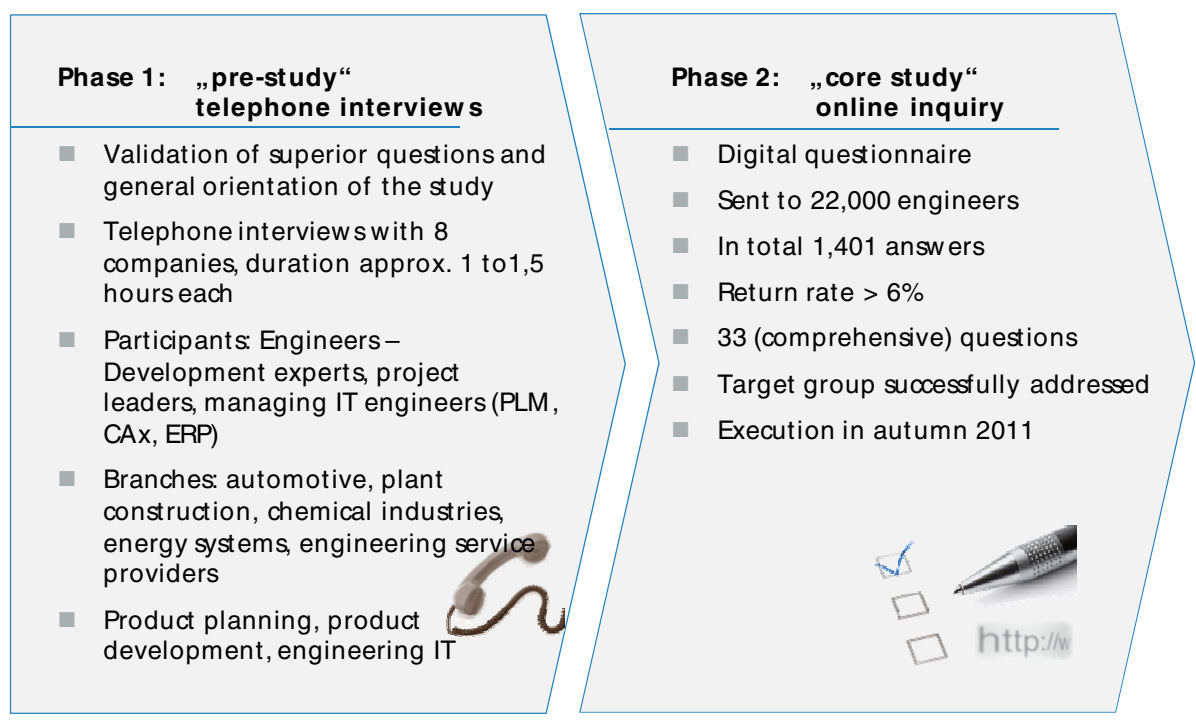

Fig. 2. Phases of the Study and Study Design

\section{Three Steps of Data Analysis and Collaboration Modeling}

The data analysis is separated in three steps: In step 1 (finished), all data was investigated towards response rates of the various answer items and values. This step was performed for the data with segmentation after branches, company size, roles, and engineering disciplines, cp. Figure 3. In step 2 (finished), correlations and dependencies amongst key factors have been analysed. In step 3 (started), a further data use to design a collaboration assessment model is scheduled to support further studies and process investigations in industry. Especially results from analysis step 1and some of step 2 are presented in this paper.

\begin{tabular}{|c|c|}
\hline Workplace / Labor & (Collaboration) processes \\
\hline $\begin{array}{l}\text { Time allocated to tasks } \\
\text { Assessment of workplace / } \\
\text { environment } \\
\text { Creativity, formalism, fun, } \\
\text { strain }\end{array}$ & $\begin{array}{l}\text { Communication } \\
\text { Coordination } \\
\text { Knowledge integration } \\
\text { Information logistics }\end{array}$ \\
\hline Tool usage & Future solutions \\
\hline $\begin{array}{l}\text { PDM, ERP } \\
\text { Data exchange platforms } \\
\text { E-mail and other } \\
\text { collaboration solutions }\end{array}$ & $\begin{array}{l}\text { Social networks } \\
\text { Mobile working solutions } \\
\text {... }\end{array}$ \\
\hline
\end{tabular}

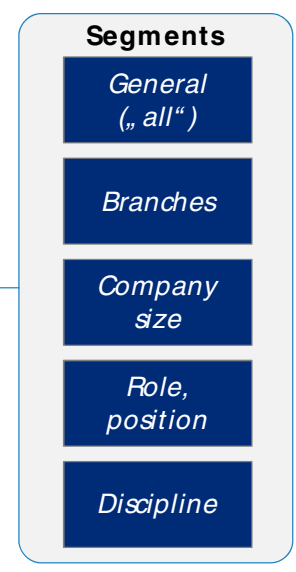

Fig. 3. Areas of further Investigation and Analyses 


\subsection{Demographic Analysis of Returned Questionnaires}

Amongst the 1,401 respondents, approximately $23 \%$ development engineers and 27\% project leaders contributed with their practical experience. Managing directors (7\%), team leaders (22\%), department leaders (13\%) and engineering practitioners from IT and process development departments $(8 \%)$ participated as well. Some represented more than one job role. More than $50 \%$ of all returning questionnaires came to almost equal proportion from the branches automotive engineering and mechanical and plant engineering. Throughout the branches aviation and aerospace, energy technologies, electrical engineering and industry, chemical and process engineering, plastics engineering, medical engineering, IT services in engineering, and engineering services there was a satisfying distribution.

\subsection{Engineering Today - Tasks, Time, and Turbulences}

A big portion of time is spent at the typical office workplace or in meeting rooms $67 \%$ of the respondents work more than $40 \%$ of working time in their office and labs. Only $6 \%$ work more than $20 \%$ of their time at home. Only 5\% work more than $20 \%$ of their working time during travelling. Even working at customer/supplier sites is more likely an exception (just $14 \%$ spend there more than $20 \%$ of their working time) - nevertheless many engineers wish to have a better support of mobile working: approx. $82 \%$ of all respondents view the possibility of to have all necessary data offline or via access through mobile devices available as a useful or very useful future vision.

Currently, the collaboration is manly happening inside the companies - between 70 and $80 \%$ answered that their internal collaboration is (very) intensive. Collaboration with partners and suppliers is less intensively performed - between 50 and $70 \%$ said that their external collaboration happens betimes or almost never. Nevertheless, almost all liked to work with partners, suppliers and customers on the same database ( $86 \%$ positive answers). According to 58\% still much product data is predominantly exchanged with customers via e-mail. This is interesting, because security concerns in opposite dominate IT policies in many companies. The possibility to digitally define and execute (engineering) workflows, which include customers, partners and suppliers, is ranked as (very) helpful by $76 \%$ of all respondents. This finding is correlating with the interview results of the pre-study: The process-oriented thinking of engineers seems to be much stronger than typically expected.

Asking for the most pressing issues, the statements from the introduction are validated: As especially wearing most engineers selected the acquisition of needed information (71\%), and the capturing of data and other routine tasks (48\%), although such tasks do not consume a large proportion of time (approx. 63\% said that this is less than $20 \%$ working time). Independent of available management solutions, just round about the haft of all participants estimated that the data necessary for their work is available in time (57\%) and in an adequate form (48\%). Especially in case of belated changes, $51 \%$ of all participants feel informed not early enough.

The majority, $87 \%$, finally thinks that Google-like tools to search in all enterprise data would be (very) helpful. A use of social networks for an exchange of engineering process and product information is still regarded skeptically. Social networks within the company are regarded as (less) useless by $60 \%$ and solutions within enterprise 
networks are regarded as (less) useless by $63 \%$. This is also a fact correlating with the pre-study. High potential for improvement is seen in the area of information logistics: Still almost all (97\%) miss a project management, which brings more transparency to responsibilities, work amount and project progression. This in turn not just applies to running projects. Many (49\%) criticize that results and experiences of earlier projects a comparably difficult to find and access. This is not astonishing, because there is hardly any time available or taken for systematic knowledge management. 58\% say that knowledge management (project reflection, lessens learnt) is done in less than $10 \%$ of working time.

Figure 4 finally is summarizing some facts mentioned. Astonishing high is the amount of communication and coordination, which is information verifying the statements in the introduction (section 1.)

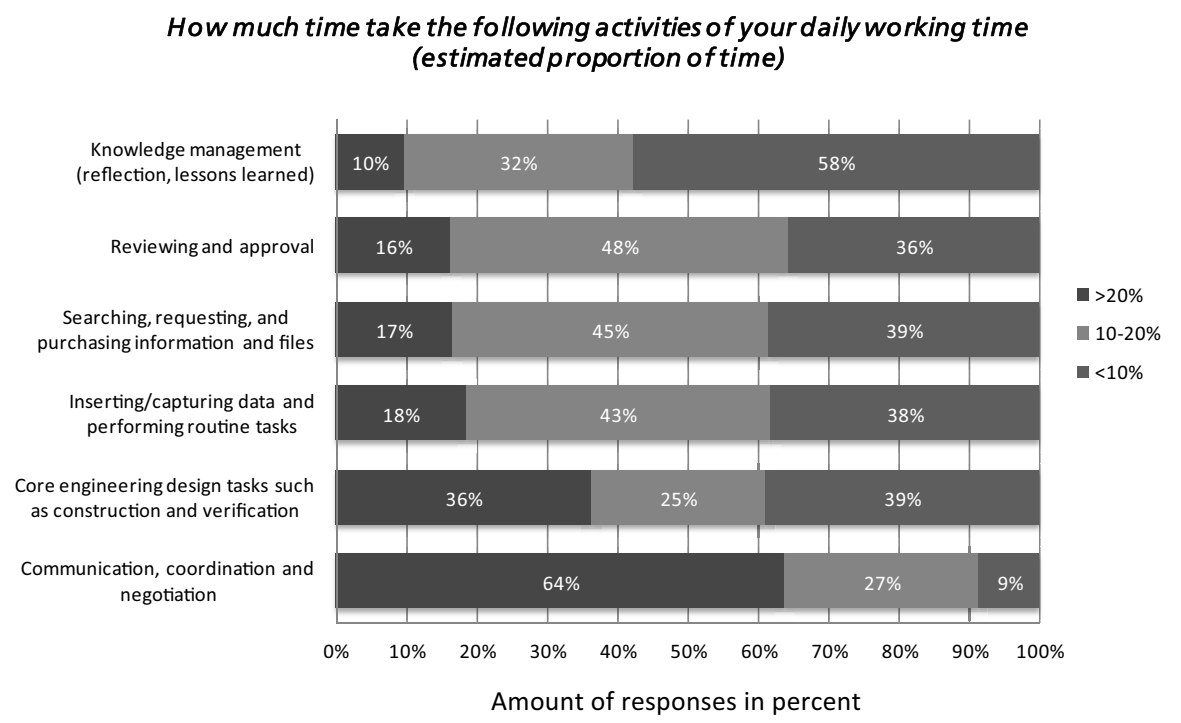

All Industries $(n=1401)$

Fig. 4. Distribution of Engineering Working Time

\subsection{PDM and ERP}

Interesting is the question how far engineers personally feel supported by PDM (Product Data Management) and ERP (Enterprise Resource Planning) systems: In the automotive segment, most feel personally well supported by PDM systems. Most also regard PDM systems as well supporting the company targets. In all other branches there is no big difference between the assessment of PDM and ERP. Figure 5 is illustrating this fact. 
The system supports me personally very well.
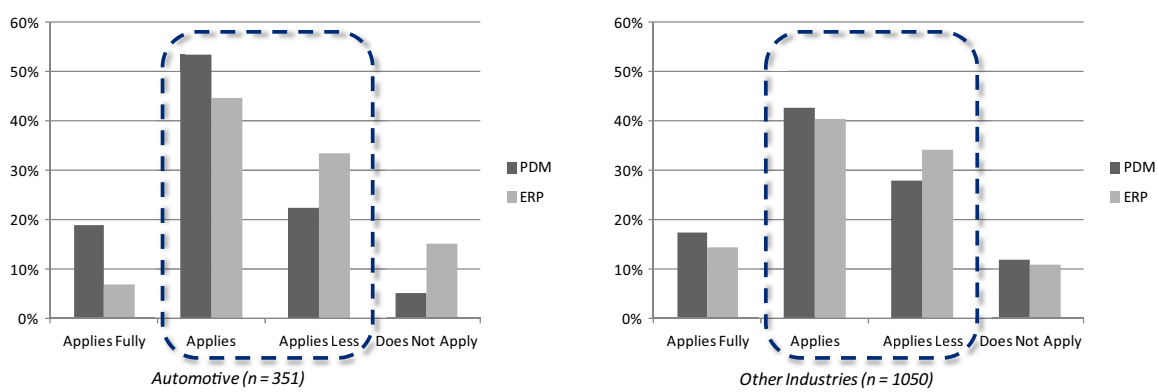

The system supports the needs of our companyo verall very well.
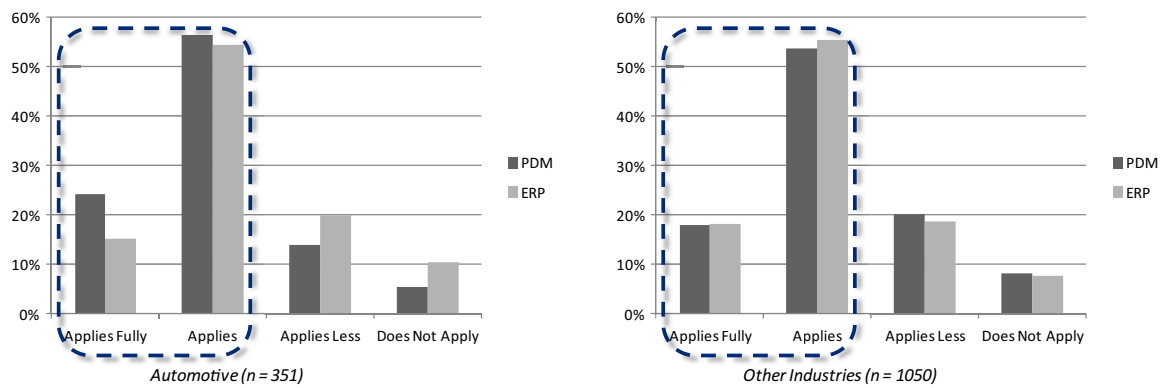

Fig. 5. Comparison of PDM and ERP Value

\section{Summary}

This study has a comparably high number of participants and is one of the largest undertaken in this area in the last decade. The distribution among branches, engineer roles, and disciplines is representing a relevant set from German industry. The findings fit to principle "feelings" about engineering practice today. Furthermore, the findings fit to other studies, for instance the study of Abramovici et al. [8]. One fact clearly corresponding is that process support (esp. for change processes) and project management support will play a major role of PDM/PLM in 2020. This has been found out in the expert study of Abramovici. In our study, this was formulated by the respondents as a kind of need and improvement potential.

"Trend technologies" such as social networks are seen skeptically. One reason, which was elicited in the pre-study, might be security concerns. Another might be missing engineering applications. Instead, typical improvement issues such as database integration and transparent process control are of major interest.

Finally, alarming is the small amount of working time, which is available for core engineering tasks, cp. Figure 4. This is clearly an issue which needs to be addressed by better process control and software support. 


\section{$5 \quad$ Prospects}

\subsection{Collaboration Capability Model}

To realize a collaborative product development process, there are primarily aspects of communication, coordination, integration of knowledge and information logistics necessary $[3,5,10,11]$. Communication is the exchange of data, information and knowledge and also includes cultural factors. Coordination includes the traditional project management tasks and determines the allocation of tasks to actors with view to the super-ordinate objective. Knowledge integration enables the collaboration of actors with different professional backgrounds by using shared mental models, metaphors and analogies [11]. Finally, information logistics is about delivering the required and understood information for the actors. The information must be available in the right quality, in the right quantity, at the right time, and at the right place [5].

The study delivered valuable information to understand the four dimensions cooperation, coordination, knowledge integration (engineering process knowledge, project knowledge, product knowledge), and information logistics in more detail. One further target is now to integrate this finding in a model to assess Collaboration Capability (cp. section 2 and analysis step 3 of the study design). A first draft of this model has already been defined and will be detailed and evaluated in further research.

\subsection{Compilation of the Study Report}

A conference paper is much too short for a comprehensive presentation of the study results. Anyhow, the data analysis is about to be finished. All findings and relevant illustrations are currently being compiled in a study report, which can be ordered via the partners of the study.

\subsection{Continuation of the Study}

The study probably will be repeated on a national level in Germany in the next years. New partners are currently motivated to join the study on an international level. The challenging task is to scale up the study and to synchronize cross-country work.

\section{References}

1. Stark, R., Krause, F.-L., Kind, C., Rothenburg, U., Müller, P., Hayka, H., Stöckert, H.: Competing in Engineering Design - the Role of Virtual Product Creation. CIRP Journal of Manufacturing Science and Technology 3, 175-184 (2010)

2. Ehrlenspiel, K.: Integrierte Produktentwicklung: Denkabläufe, Methodeneinsatz, Zusammenarbeit (4., aktualisierte Aufl.). Hanser, München (2009)

3. Eigner, M., Stelzer, R.: Product Lifecycle Management: Ein Leitfaden für Product Development und Life Cycle Management. Springer, Heidelberg (2009)

4. Gausemeier, J.: Vernetzte Produktentwicklung: Der erfolgreiche Weg zum Global Engineering Networking. Hanser, München (2006) 
5. Kern, E.-M.: Verteilte Produktentwicklung - Rahmenkonzept und Vorgehensweise zur organisatorischen Gestaltung. GITO-Verl., Berlin (2005)

6. Lu, S.C.-Y., Elmaraghy, W., Schuh, G., Wilhelm, R.: A scientific Foundation of Collaborative Engineering. CIRP Annals - Manufacturing Technology 56(2), 605-634 (2007)

7. Willaert, S.S.: Collaborative engineering: A case study of concurrent engineering in a wider context. Journal of Engineering and Technology Management 15(1), 87-109 (1998)

8. Abramovici, M., Bellalouna, F., Neubach, M.: Delphi Studie PLM 2020, Experteneinschätzungen zur zukünftigen Entwicklung des Lifecycle Managements. ITM, RuhrUniversität Bochum (2009)

9. RAAD Research: Product Lifecycle Management Deutschland 2012, PLM-Markt 2012, Integrierte Lösungen vs. offene Systeme (Study). Hoppenstedt Firmeninformationen GmbH - Geschäftsbereich RAAD Research (2011)

10. Seiffert, U.: Virtuelle Produktentstehung für Fahrzeug und Antrieb im Kfz: Prozesse Komponenten Beispiele aus der Praxis. ATZ/MTZ-Fachbuch, Vieweg + Teubner, Praxis, Wiesbaden (2008)

11. Steinheider, B.: Supporting the co-operation of R\&D-teams in the product development process. In: Proceedings of the 5th Conference on Engineering Design and Automation, Las Vegas, Nevada (2001) 